\title{
Short-latency discriminative unit response: Engram or bias?
}

\author{
MICHAEL GABRIEL \\ University of Texas at Austin, Austin, Texas 78712
}

\begin{abstract}
Recent studies have shown changes during conditioning in CS-related neuronal activity. The changes were associative in character, similar to behavioral changes which result from conditioning. However, unlike behavioral conditioned responses, the neuronal responses had very short latencies $(5-35 \mathrm{msec})$. In the present paper, two hypotheses accounting for the short-latency associative activity are examined. One hypothesis assumes acquisition of a neuronal modification (engram) in the vicinity of the recording electrode. The second hypothesis postulates a tonic flow of impulses originating from a distant neural locus, which biases the activity of the recorded neurons to produce the associative response. Implications of each viewpoint are examined and the greater appeal of the bias hypothesis is asserted, based upon certain implausible implications of the engram hypothesis. Recently obtained data are presented which favor the bias hypothesis over the engram hypothesis for one CNS locality.
\end{abstract}

In recent years there has been great interest in the study of neuronal correlates (single and multipleunit) of learning in awake behaving animals (see reviews of John, 1967; Kandel \& Spencer, 1968; Leiman \& Christian, 1973; Thompson, Patterson, \& Teyler, 1972). This article is concerned with one particular finding from some of these studies. It is the observation of associative (learned) responses displayed by CNS neurons to stimuli used as CSs for conditioning. An example is the data of Olds and his colleagues (Kornblith \& Olds, 1973; Olds, Disterhoft, Segal, Kornblith, \& Hirsh, 1972) for neurons of various CNS regions in the rat, and by Gabriel and his colleagues (Gabriel, Miller, \& Saltwick, 1976, in press; Gabriel, Satlwick, \& Miller, 1975; Gabriel, Wheeler, \& Thompson, 1973a, b) for neurons of the rabbit anterior cingulate cortical region and the rabbit medial geniculate nucleus. In these studies, neuronal activity showed the associative characteristic of stimulus control, i.e., greater frequency of firing to the CS used for conditioning compared to stimuli not paired with reinforcement. These studies had appropriate controls to eliminate nonassociative interpretations of the data. ${ }^{1}$ Most importantly, the studies showed that the neuronal effect occurs with very short latency (5-35 msec) measured from stimulus onset.

Here, our interest is with a particular interpretation of such findings, specifically, the one presented by Olds et al. (1972). Their interpretation

The author wishes to thank Dr. Dennis McFadden for insights and critical comments essential to the manuscript. The research results presented in this paper were supported by grants from the Biomedical Sciences Committee, the Spencer Foundation, and by National Institute of Mental Health Grant MH 26276-01. is stated with considerable precision, so that a number of implications become immediately evident with regard to the nature and direction of future research on the neurophysiological basis of learning. However, implications are only as valid as their generative assumptions. The purpose of the present paper is to show that the initial assumptions of Olds et al. (1972) are not necessary or compelling regarding the significance of short-latency associative neuronal reactions. An alternative set of assumptions and supporting data will be provided, and the alternative implications for future research, spelled out.

Olds and his co-workers asserted that the neuronal latencies observed in their study were so short that there was not sufficient time for synaptic transmission or conduction over appreciable distances within the CNS. Therefore, short-latency associative responses to the CS could not first occur at a CNS locus distant from the locus of recording, only later to arrive at the locus of recording. Rather, they must be direct effects of the stimulus, totally uninfluenced by stimulus-evoked inputs relayed from other parts of the brain.

Since there is insufficient time for a neural influence to be relayed from a great distance, such an influence cannot account for the short-latency activity of the neuron. It follows that the responding neuron must contain within itself, or very near to itself, a mechanism sufficient to mediate the associative response. The mechanism is a localized structural or metabolic change of neurons acquired during conditioning. It may be identified as a member of one of the classes denoted by the concept, "engram." Thus, by the theory under consideration, an engram underlying the neuronal activity must exist in a location which is within or very close to the recorded neuron, and the neuronal activity functions to indicate 
the locus of the engram. In the words of Olds and his co-workers, the neuronal activity is "at the site of the mnemonic record" (Olds et al., 1972).

The phrase, "engram hypothesis," will be used in the remainder of this paper to denote this interpretation. A primary implication of the engram hypothesis is that one may "map" the neuroanatomical loci of engrams in the brain by noting all points which display a short-latency associative effect. Ultimately, this argument paves the way for a powerful approach to the study of neuroanatomical specificity in learning.

The precise nature of the molecular mechanism whereby engrams are formed is not at issue here. Many models have been proposed to explain their formation (e.g., Burke, 1966; Pfaff, 1969). These are all equivalent for purposes of the present discussion, in that they all postulate local changes in the CNS to result from interactions during conditioning among a small number (2-5) of cells. What is at issue is the validity of the idea that engrams invariably underlie short-latency neuronal reactions.

The engram hypothesis fosters an optimistic outlook on the potential fruitfulness of mapping the CNS for learning-related structural change. Thus one may easily wish that the hypothesis were true. Unfortunately, certain considerations show that caution is called for in interpreting short-latency responses solely in terms of the engram hypothesis. The latter viewpoint is not the only one able to account for such responses. Moreover, the engram view has implications which lead to implausible predictions. These do not seem likely to receive empirical support.

Of crucial importance to the engram hypothesis is the fact that there is insufficient time for shortlatency responses to be affected by prior neuronal events in the interval beginning at CS onset. Thus, a central premise of the engram hypothesis is that short-latency responses are not relayed from distant regions which previously responded to the CS. This premise seems an essentially sound one. However, the engram hypothesis uses this premise to assert a corollary proposition which is not sound. It is the idea that short-latency responses occur without any influence from distant regions of the CNS. From this, it follows that one may "map" the CNS for localized engrams by noting points which yield shortlatency responses. Moreover, the corollary proposition implies that short-latency responses occur to the absolute, not relative, properties of the incoming stimulus. $^{2}$

A major problem with the corollary proposition and its implications is that they are inconsistent with the well-established principle that stimulus control of a learned response is exerted by the context of the learning situation, as well as by specific response- signaling cues. This point may be illustrated in a variety of ways. For example, postconditioning exposure of subjects to the conditioning apparatus, in the absence of the CS, produced complete extinction of behavioral CRs (Gabriel, 1970; Gabriel \& Vogt, 1972). If contextual stimuli exert control over learned behavioral responses, why then should not contextual stimuli also influence conditioned neuronal responses? It seems reasonable to assume that the mechanisms which mediate short-latency neuronal reactions are context-dependent mechanisms. As such, they would take on different properties in each situation encountered by an organism. Contextual stimuli affect severai sensory modalities. Thus, the properties appropriate to a given context would be signaled to a given CNS locality via several sensory pathways, and inputs from several regions of the brain would govern short-latency reactions. The alternative assumption, that engrams dedicate neurons to exclusive control by specific isolated stimuli, is unpalatable at best. It seems remote indeed that a short-latency conditioned neuronal response would occur to the absolute properties of a discrete CS outside of the environment in which conditioning took place. Yet this represents one of the experimental results which would be required to provide preferential support of the engram hypothesis.

The problems referred to are consequent upon the adoption of the engram hypothesis. The purpose of the present paper is to point out that there exists an alternative to the engram hypothesis. The alternative hypothesis, like the engram hypothesis, accounts for associative reactions with short latency. Yet, the alternative view is preferable because it avoids the implausible features of the engram hypothesis.

Assume that some neurons are tonically influenced to respond to some inputs and not to others. In other words, assume that some neurons are continuously bombarded by synaptic inputs, and that these inputs bias the neurons to respond to significant stimuli, and to "ignore" nonsignificant stimuli.

Bias may be assumed to operate upon single neurons, but it need not originate within or nearby them. Instead, it may be carried to a given neuron over a considerable distance along a path which is separate from the one bearing information about the stimulus. The centrifugal pathways which mediate neural modulatory effects at relatively peripheral levels of certain sensory systems (e.g., Granit, 1955; Winter, 1965) represent examples of anatomical organization appropriate to the bias hypothesis.

The idea of bias is a relatively general one; it is represented by a variety of specific hypothetical mechanisms. One example is the selective filtering mechanism believed by some investigators (e.g., 
Hernández-Peón, Scherrer, \& Jouvet, 1956) to operate upon neural transmission in afferent pathways, and to underlie the behavioral phenomena of selective attention.

On the other hand, a more general notion than bias, which subsumes the latter, is the idea of "set," or the totality of sustained responses to contextual stimuli (e.g., apparatus stimuli) of a learning situation. It is instructive to consider bias as a submechanism of set, because in so doing, one appreciates the sustained effects of bias. That is, bias exists tonically in a sustained fashion before, during, and after the presentation of a discrete signal (e.g., CS). Just as a filtered electronic circuit, a neural conduction pathway with bias remains biased whether or not a signal is present. Clearly the spirit of the bias hypothesis is compatible with the welldemonstrated controlling effects of contextual cues upon conditioned responses.

It is important to note that the involvement of the concept of set does not mean that the shortlatency neuronal responses are not learned responses. Insofar as they conform to the criteria of associative learning, they are just as legitimately associative in character as behavioral conditioned responses. Moreover, set itself is a learned effect assumed to be conditioned to and evoked by contextual stimuli of the learning situation.

An important consequence of the bias hypothesis is that one is disinclined to use short-latency acquired unit responses to "map" the neuroanatomical loci of structural (learning) changes. This is true because the tonic bias mediating an associative neuronal response may originate at a CNS locus distant from the locus of the responding neuron. Thus, the locus of the latter need not be the same as the locus of an engram.

One question which should be raised is the question of whether there are any reasons other than theoretical ones favoring the bias hypothesis over the engram hypothesis. Recall the centrifugal neuroanatomical pathways which mediate modulation of afferent neural activity. These pathways support the bias hypothesis in that they illustrate possible anatomical bases of the biasing mechanism, but they do not compel acceptance of the hypothesis. To demonstrate bias, one must first show neural activity in a behaving subject which is unequivocally associative in nature. In addition, one must demonstrate that the associative neuronal activity is dependent upon the influence of some (presumably centrifugal) conduction pathway other than the afferent path to the responding neuron. Unfortunately for the bias hypothesis, the second of these two effects has not been demonstrated.

Recent observations from our own laboratory do not meet all of the requirements for a demonstration of biasing, but they do provide a posteriori support of the bias hypothesis over the engram hypothesis (Gabriel, Miller \& Saltwick, 1976; Gabriel, Saltwick, \& Miller, 1975). In these studies, we observed differential conditioning and reversal of short latency multiple-unit activity in the medial geniculate nucleus (MGN) of the rabbit. These changes occurred in parallel with acquisition and reversal of a discriminative behavioral (avoidance) CR.

The observation of reversal of the acquired neuronal response eliminated possible nonassociative interpretations of the data. Moreover, our effect appeared to be a prime candidate for explanation by the bias hypothesis. This is true because of the rapid switching seen on early sessions of reversal training between the neuronal response appropriate to acquisition and that appropriate to reversal (Figure 1). Specifically, either abrupt loss or reversal of the discriminative neuronal activity appropriate to original acquisition occurred in the initial sessions of reversal training. Speaking anthropomorphically, these abrupt neuronal changes suggested that the subjects quickly switched off or reversed the listening strategy of acquisition, in response to the occurrence of extra shocks received on the early sessions of reversal. Since the subjects adopted the safe strategy of responding behaviorally to both $\mathrm{CS}+$ and $\mathrm{CS}-$ on subsequent sessions, relatively few shocks occurred on these sessions and the listening strategy (MGN neuronal response) reverted in some subjects to the dominant one used in the previous (acquisition) task. Only after considerable additional experience with the reversal task, were both behavioral and neuronal (attentional?) reversal achieved. The critical point is that it seems more reasonable to account for the sudden switches of the differential neuronal response in terms of a rapid switching of the pattern of centrifugal flow into MGN than in terms of a rapid manufacture, dissolution, and remanufacture of structures (engrams) within MGN. The return during reversal training of the MGN response appropriate to the acquisition task is especially difficult for the engram hypothesis, since the latter must account for reformation of an "old" engram in the presence of differential reinforcement appropriate to the opposite ("new") engram. The centrifugal flow assumed by the bias hypothesis to account for these effects may have occurred over one of the recently documented corticofugal projections to MGN (Pontes, Reis, \& Sousa-Pinto, 1975; Watanabe, Yanagisawa, Kanzaki, \& Katsuki, 1966).

The reader may argue that the bias hypothesis lacks parsimony. It pushes the problem of structural change off to some remote and unspecified region of the CNS. Moreover, the kind of structural changes required in 

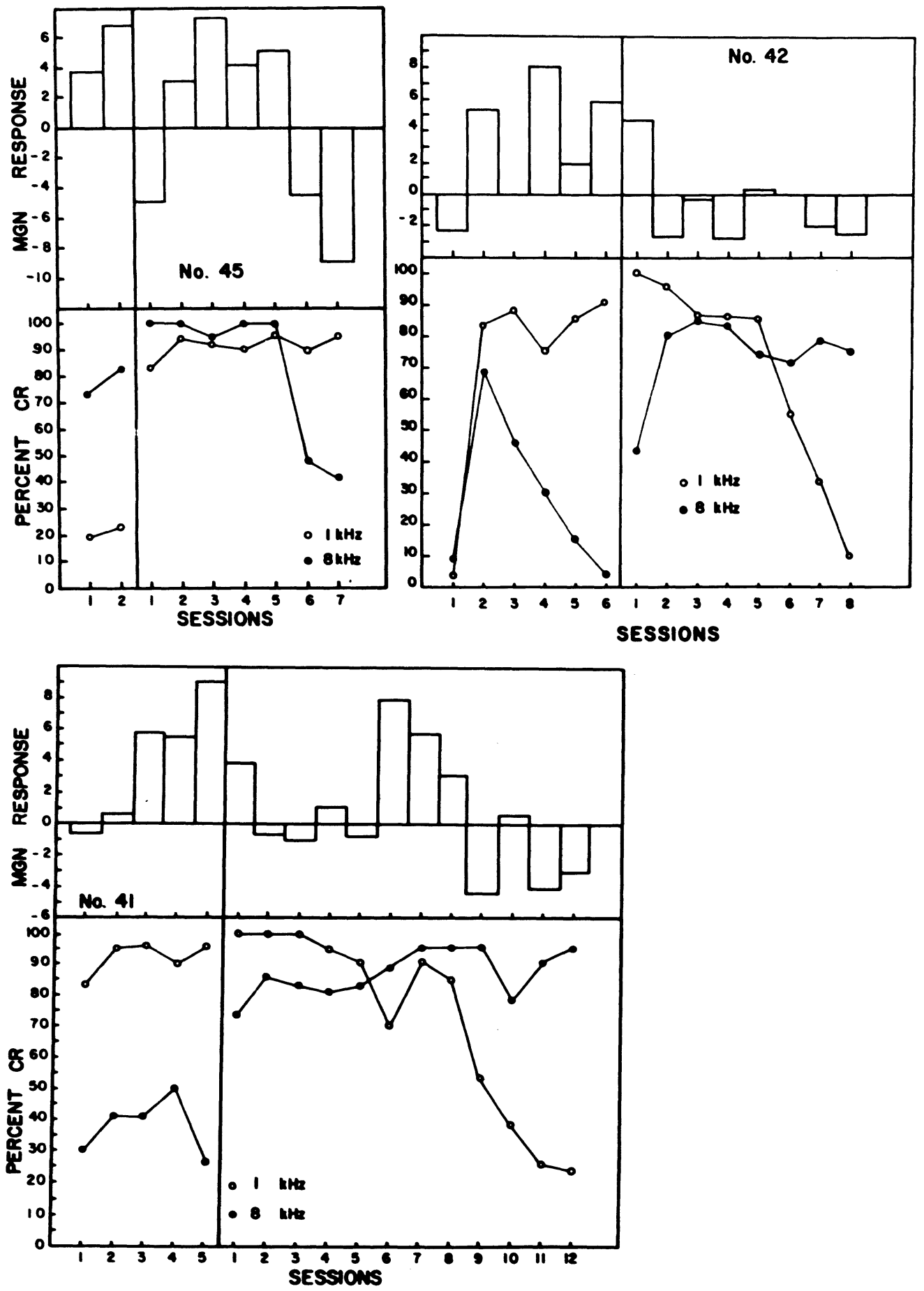

Figure 1. The graphs shown here illustrate the relationships between neuronal (MGN) and behavioral response during differential conditioning (left or vertical lines) and reversal (right of vertical lines) in three rabbits. The lower portion of each graph shows the percentage of behavioral responses to the CS+ and the CS- over consecutive daily sessions. The CS+ was either an 8-kHz tone (•) or a 1-kHz tone (O). The upper portions of the graph show the magnitude of the MGN differential response [CS+ (acquisition) - CS(acquisition)] in each session. The scores were based on the 10-msec period (bin) after CS onset, which showed the greatest crossover in terminal reversal relative to terminal acquisition. The same bin was used throughout the analysis of a given subject's data. Bins 5,5 , and 6 were used for rabbits $41,42,45$, respectively. Virtually identical results were obtained when the average value of five bins surrounding the single bin shown here were used. Each bar shows the magnitude of the differential response (CS+ - CS-) minus the magnitude of this difference obtained during pretraining with tones plus noncontingent shock. Bars above the baseline in acquisition reflect a greater response to the acquisition CS+ than to the acquisition CS-. Bars below the baseline in reversal indicate a greater response to the reversal CS+ than to the reversal CS-. These data illustrate abrupt crossover just after the onset of reversal training, followed by a return to the response appropriate to acquisition training, and a change back to the reversal pattern, at the end. Further details of these results are presented by Gabriel, Miller, and Saltwick (1976). 
these remote regions would have to be very complex indeed if they are to account for the switching of listening strategies which was suggested by our data.

Fortunately for the bias hypothesis, these arguments are not compelling. The ability to switch listening strategies is in all liklihood an ability which is analogous to, say, locomotion or digital control. One could assume that considerable neural circuitry for these abilities is prewired, that the learning required to actualize this circuitry takes place early in development, and that control over listening strategy is well overlearned in the adult. The problem facing a subject in conditioning is thus not that of learning from scratch to produce a listening strategy, but rather, that of learning which of many possible wellpracticed strategies to adopt in a given situation. The signaling of an appropriate prewired listening strategy would be considerably less complex than de novo learning of the strategy. It is also compatible with the abrupt switching of MGN filtering which is suggested by our results to occur in the initial sessions of reversal training.

This argument simplifies the informational "load" placed upon structural engrams. Nevertheless, it is clear that some structural change is necessary to accomplish the relatively simple signaling of appropriate listening strategies. It is tempting to speculate that structural changes (engrams) account exclusively for the ability of contextual stimuli to elicit appropriate sensory and motor biases, and that the biases, in turn, account exclusively for short-latency associative reactions.

Clearly, the bias hypothesis argues against the idea of mapping the brain for short-latency neuronal responses in order to localize engrams. However, the implications of bias are not uniformly negative with respect to possible electrophysiological analyses of neuroanatomical factors in learning. Rather, the bias hypothesis causes a shift in emphasis away from localization of engrams toward a search for the CNS systems which mediate tonic biasing effects. Specifically, the bias hypothesis fosters a search for neural systems which show a tonic associative response to contextual properties of the learning situation, which have neuroanatomical projections to the regions manifesting short-latency associative reactions, and which can be shown to bear a necessary relationship to the short-latency responses.

This analysis stresses the importance of tonic associative activity in the CNS. Thus, one may raise a question regarding the usefulness of research which focuses on phasic short-latency neuronal responses. If these cannot be used to localize engrams, then what good are they? In fact, the study of short-latency effects is an essential component of electrophysiological analyses of brain function relevant to conditioning.
As Olds has pointed out, short-latency reactions are not relayed from other brain regions, or from incipient effector activity. Thus they yield a picture of activity specific to the recorded locus not confounded by inputs from more quickly responding regions. In the absence of mediation from other regions, tonic bias or local engram would seem the major feasible alternatives remaining to account for short-latency responses. ${ }^{3}$ Thus, the discovery of sites yielding short-latency responses sets the stage for subsequent analyses. The subsequent analyses may establish the origins of tonic bias. If exhaustive search fails to reveal tonic effects, this would suggest that local engrams account for the short-latency response, and microbiophysical analyses may reveal the engrams.

A second point should be made with respect to the usefulness of short-latency reactions. Because these reactions are not relayed from distant loci, they should be expected to yield information about the function of neural transmission at the recorded loci. Results of recent studies have supported this expectation by showing distinctive neuron-behavior relationships in different brain regions. For example, the short-latency response of neurons of rabbit limbic cortex and anterior thalamus shows acquisition of an original discrimination, increased magnitude of the original discrimination on the initial sessions of reversal training, and no clear reversal effect at the end of reversal training (Gabriel, Miller, \& Saltwick, in press). This outcome is to be contrasted with the discriminative activity of MGN, as reported above. The MGN showed abrupt equalization or reversal of the original discrimination at the onset of reversal training, and a clear reversal effect at the end of reversal training.

A detailed discussion of these findings is contained in the original reports. For the present purpose, it is sufficient to point out the unique relationship to behavioral performance shown by the neuronal activity in different regions of the CNS. The implication is that each region contributed uniquely to behavioral output. Thus, it would appear that short-latency reactions provide information on the distinctive behavioral relevance of activity in different parts of the brain.

One final point. It is necessary to mention that Olds and his colleagues have recognized the bias hypothesis, and have attributed importance to it (e.g., Disterhof \& Olds, 1972; Kornblith \& Olds, 1973) relative to the position which they espoused in Olds et al., (1972). Only the latter article places virtually exclusive emphasis on the engram argument. The purpose of the present paper is not to criticize their obviously excellent work but primarily to indicate that the loci of engrams may not be identified with loci yielding short-latency associative responses. 


\section{REFERENCE NOTE}

1. Olds, J. Presentation given at the workshop on neural organization forlearning. Winter Conference for Brain Research, January 1976, Keystone, Colorado.

\section{REFERENCES}

BuRkE. W. Neuronal models for conditioned reflexes. Nature, 1966. 5033. 269-271.

Disterhoft, J. S., \& Olds, J. Differential development of conditioned unit changes in thalamus and cortex of rat. Journal of Neurophysiology, 1972, 35, 665-679.

GABRIEL, M. Intersession exposure to conditioning apparatus, avoidance extinction and intertrial behavior. Journal of Comparative and Physiological Psychology, 1970, 72, 244-249.

Gabriel, M.. Miller, J. D., \& Saltwick, S. E. Multiple unit activity of the rabbit medial geniculate nucleus in conditioning, extinction, and reversal. Physiological Psychology, 1976, 4, 124-134.

Gabriel, M., Miller, J. D., \& Saltwick, S. E. Unit activity in cingulate cortex and anteroventral thalamus of the rabbit during differential conditioning and reversal. Journal of Comparative and Physiological Psychology, 1976, in press.

Gabriel, M., Saltwick, S. E., \& Miller, J. D. Conditioning and reversal of short latency multiple-unit responses in the rabbit medial geniculate nucleus. Science, 1975, 189, 1108-1109.

GABRIEL, M.. \& VOGT, J. Incubation of avoidance CR's in the rabbit produced by increase over time in stimulus generalization to apparatus. Behavioral Biology, 1972, 7, 113-126.

Gabriel, M., Wheeler, W., \& Thompson, R. F. Multiple-unit activity of the rabbit cerebral cortex in single-session avoidance conditioning. Physiological Psychology, 1973, 1, 44-45. (a)

Gabriel. M., Wheeler, W.: \& Thompson, R. F. Multiple unit activity of the rabbit cerebral cortex during stimulus generalization of avoidance behavior. Physiological Psychology, 1973, 1, 313-320. (b)

Granit, R. Receptors and sensory perception. New Haven: Yale University Press, 1955.

Hernández-Peón, R., Scherrer, H., \& Jouvet, M. Modification of electrical activity in cochlear nucleus during "attention" in unanesthetized cats. Science, 1956, 123, 331-332.

John. E. R. Mechanisms of memory. New York: Academic Press, 1967.

Kandel, E. R., \& Spencer, W. A. Cellular neurophysiological approaches in the study of learning. Physiology and Behavior, $1968,48,65-134$.

KERR, D. I. B.. \& HAGBARTh, K. E. An investigation of olfactory centrifugal fiber system. Journal of Neurophysiology, 1955, 18 , $362-374$.

KoRnblith, C., \& Olds, J. Unit activity in brain stem reticular formation of the rat during learning. Journal of Neurophysiology, 1973, 36, 489-501.
Leiman, A. L., \& Christian, C. N. Electrophysiological analyses of learning and memory. In J. A. Deutsch (Ed.), The physiological basis of memory. New York: Academic Press, 1973.

Olds, J., Disterhoft, J., Segal, M., Kornblith, D. L., \& HiRsh, R. Learning centers of rat brain mapped by measuring latencies of conditioned unit responses. Journal of Neurophysiology, 1972, 35, 202-219.

PfafF, D. Parsimonious biological models of memory and reinforcement. Psychological Review, 1969, 76, 70-81.

Pontes, C., Reis, F. F., \& Sousa-Pinto, A. The auditory cortical projections onto the medial geniculate body in the cat. An experimental anatomical study with silver and autoradiographic method. Brain Research, 1975, 91, 43-63.

Thompson, R. F., Patterson, M. M., \& Teyler, T. F. The neurophysiology of learning. Annual Revien of Psychology, 1972. 23. 73-104.

Watanabe. T., Yanagisawa, K., Kanzaki, J., \& Katsuki, T. Cortical efferent flow influencing unit responses of medial geniculate body to sound stimulation. Experimental Brain Research. 1966, 2. 302-317.

Winter, D. L. N. Gracilis of cat: Functional organization and corticofugal effects. Journal of Neurophysiology, 1965, 28, 48-70.

\section{NOTES}

1. Recently, Olds has reported the possibility that a cyclic factor related to the regular occurrence of reinforcement may have contributed to the short-latency reactions in his studies. Thus the claim that they were cue-specific associative reactions was called into question. However, cue-specific short-latency neuronal responses were demonstrated in some brain regions even when the cyclic factor was eliminated (Olds, Note 1).

2. The corollary proposition is not explicitly stated by Olds and his colleagues (Olds et al., 1972). However, it is fully implicit in their contertion that one may map for localized engrams by noting the points which yield short-latency responses.

3. There is a third alternative able to account for the shortlatency reactions. This alternative is essentially a combination of the tonic bias hypothesis and the engram hypothesis. It states that local engrams account for short-latency reactions, but in order to do so the engrams must be activated by a tonic input other than the afferent input to the recorded neuron. Thus many different engrams exist in a given locality (say, MGN), but a distinctive tonic input is necessary for a particular subset of these to exert an effect on neuronal activity. Detailed consideration of this possibility was not given in the present paper because it has less parsimony than the tonic bias and local engram hypotheses, and because the combination hypothesis seems difficult to rationalize in terms of established principles of cellular neuronal interaction. Moreover, the combination hypothesis calls for the same experimental approach as that called for by the tonic bias hypothesis.

(Received for publication December 1, 1975; revision accepted March 3, 1976.) 